Réglementation

\title{
Supervision réglementaire des agents pathogènes pour les humains et des toxines au Canada
}

\author{
Labrie $C^{1 *}$, Lecordier $\mathbf{S}^{1_{*}}$ \\ ${ }^{1}$ Centre de la biosûreté, Agence de la santé publique du Canada, Ottawa (Ontario) \\ *Correspondance : cinthia.labrie@canada.ca, sonia.lecordier@canada.ca
}

\section{Résumé}

De 1994 à 2009, la supervision des agents pathogènes humains et des toxines par le gouvernement fédéral se limitait aux installations qui importaient ces substances dans le cadre du Règlement sur l'importation des agents anthropopathogènes. Ce champ de compétence étroit restreignait la capacité du gouvernement canadien à réglementer et surveiller un large éventail d'activités, notamment celles faisant intervenir des agents pathogènes humains et des toxines provenant de domestiques. En 2009, la Loi sur les agents pathogènes humains et les toxines (la $L o l$ ) a reçu la sanction royale pour établir un régime national de sécurité et de sûreté et élargir la supervision par un processus national normalisé visant à vérifier que les agents pathogènes pour les humains et des toxines soient utilisés de manière sûre et sécuritaire.

La Loi et le Règlement sur les agents pathogènes humains et les toxines (le Règlement), en vigueur depuis le $1^{\mathrm{er}}$ décembre 2015, établissent désormais les exigences légales et réglementaires pour la supervision globale du contrôle des agents pathogènes pour les humains et des toxines au Canada. L'élargissement de la réglementation et les activités du programme de surveillance ont pour but de réduire les risques posés par les agents pathogènes humains et les toxines et de renforcer les systèmes de gestion de la biosécurité qui servent à protéger la santé des Canadiens.

\section{Introduction}

Depuis 2001, de nombreux accidents et incidents de laboratoire de haute visibilité ont eu lieu à l'échelle nationale et internationale, notamment des expositions, des infections contractées en laboratoire et des délits intentionnels associés aux des agents pathogènes humains et aux toxines. Par exemple, en octobre 2001, des lettres contenant de l'anthrax ont été envoyées à diverses personnes aux États-Unis, entraînant cinq décès (1). Environ quatre mois après la fin de l'éclosion de syndrome respiratoire aigu sévère (SRAS) en 2003, un étudiant en microbiologie de Singapour a contracté le SRAS en raison de manquement dans les protocoles et les procédures de biosécurité (2). En 2011, une éclosion de 109 cas de Salmonella typhimurium associée à des laboratoires de microbiologie cliniques et d'enseignement a touché 38 États des États-Unis (3).

Compte tenu des exigences limitées et variables régissant la déclaration de ces incidents au niveau international, ainsi que des définitions diverses de ce qui constitue un incident, il est probable que ces événements continueront à être sous-déclarés.

Comme les autres pays, le Canada est vulnérable à la libération accidentelle ou intentionnelle ou à l'utilisation malveillante d'un agent qui pourrait provoquer des perturbations sociales et économiques considérables et avoir des répercussions à l'échelle internationale. Qu'ils soient situés en milieu universitaire, dans les hôpitaux, dans les ministères gouvernementaux ou dans le secteur privé, de nombreux laboratoires canadiens travaillent avec des agents pathogènes humains et des toxines, comme la bactérie Escherichia coli, le virus de la grippe A et la Listeria. S'ils ne sont pas manipulés correctement, ces agents peuvent provoquer une maladie modérée, voire la mort. Les répercussions en matière de santé 
publique peuvent être importantes et comprennent un risque d'éclosion dans la collectivité, dans le pays ou même à l'étranger. Les agents pathogènes humains et les toxines peuvent également présenter des risques pour la sécurité et l'économie nationales en cas de vol, de bioterrorisme ou de création d'agents pour lesquels il n'existe aucune contre-mesure.

Depuis le début des années 2000, la communauté internationale prend conscience de la nécessité de renforcer la supervision nationale de l'utilisation des substances biologiques. De nombreux pays progressent vers un contrôle plus strict de l'éventail des activités faisant intervenir des agents pathogènes humains et des toxines, telles que l'importation, la possession, l'utilisation, l'entreposage, le transport, l'élimination et l'exportation.

Avant 2009, la supervision des agents pathogènes humains et des toxines par le gouvernement fédéral se limitait aux installations qui importaient ces substances dans le cadre du Règlement sur l'importation des agents anthropopathogènes, ce qui empêchait le gouvernement canadien de vérifier que les agents pathogènes humains et les toxines acquis de sources domestiques étaient utilisés de manière sûre et sécuritaire.

La Loi sur les agents pathogènes humains et les toxines (la Loi) (4) a reçu la sanction royale en 2009. Cette loi a pour objet d'établir un système de supervision visant à protéger la santé et la sécurité du public contre les risques que présentent les agents pathogènes humains et les toxines, qu'ils proviennent de sources situées dans le pays ou qu'ils aient été importés au Canada. L'Agence de la santé publique du Canada (l'Agence) est l'autorité nationale compétente en matière de biosûreté et de biosécurité des agents pathogènes humains et des toxines, et elle est responsable de leur réglementation en vertu des pouvoirs qui lui sont conférés par la Loi et par le Règlement sur les agents pathogènes humains et les toxines (le Règlement) (5).

Le Règlement est entré en vigueur le $1^{\text {er }}$ décembre 2015. Les laboratoires et les autres parties réglementées doivent désormais présenter une demande de permis pour exercer les activités contrôlées, notamment avoir en sa possession, manipuler, utiliser, produire, entreposer, permettre à quiconque d'y avoir accès, transporter, importer, exporter, rejeter, abandonner et éliminer un agent pathogène humain ou une toxine. II s'agit d'un cadre d'octroi de permis fondé sur le risque, visant à améliorer la supervision par le gouvernement fédéral des agents pathogènes humains et des toxines au Canada, à établir des exigences à l'échelle nationale pour la manipulation sûre et sécuritaire d'agents pathogènes humains et de toxines qui s'appliquent à toutes les installations exerçant des activités contrôlées avec ces agents, et à garantir que les personnes qui ont accès à une liste établie d'agents pathogènes humains et de toxines exigeant une cote de sécurité élevée (appelés agents biologiques à cote de sécurité élevée [ABCSE]) ont fait l'objet d'une évaluation et été jugées fiables et dignes de confiance.

Le présent article a pour objectif de décrire les mécanismes qui sont désormais en place en vertu de la Loi et du Règlement pour gérer les risques liés aux agents pathogènes humains et aux toxines, afin de prévenir la propagation de maladies transmissibles et de protéger la santé des Canadiens.

\section{Aperçu de la Loi et du Règlement}

La Loi et le Règlement ont pour objet d'établir un régime de sécurité et de sûreté visant à protéger la santé et la sécurité du public contre les risques que présentent les agents pathogènes humains et les toxines. Ainsi, le nouveau cadre d'octroi de permis prévoit la supervision des activités contrôlées qui sont associées à des agents pathogènes humains et des toxines, décrit les pouvoirs et les fonctions ainsi que les qualifications des agents de la sécurité biologique et établit les exigences relatives aux habilitations de sécurité. 
En 2009, certains articles de la Loi sont entrés en vigueur afin de créer une plateforme de biosûreté devant servir de fondement à l'application de la Loi. II s'agissait notamment des articles suivants :

- l'exigence, pour toute personne responsable d'activités qui sont associées à des toxines mentionnées à l'Annexe 1 de la Loi ou des agents pathogènes humains appartenant au groupe de risque 2, 3 ou 4, d'être inscrite auprès de l'Agence;

- $\quad$ l'interdiction des activités faisant intervenir des agents pathogènes humains et des toxines mentionnés à l'Annexe 5 (qui ne comprend actuellement que la varicelle) et l'obligation d'informer l'Agence de toute production par inadvertance d'agents pathogènes humains ou de toxines figurant à l'Annexe 5;

- l'obligation de prendre toutes les précautions raisonnables pour protéger la santé et la sécurité du public lorsque l'on mène sciemment des activités avec des agents pathogènes humains et des toxines. Pour aider à satisfaire cette obligation en vigueur, il est recommandé d'être en conformité avec la Norme canadienne sur la biosécurité (6).

La plupart des dispositions relatives aux infractions et aux sanctions sont désormais en vigueur, de même que les pouvoirs d'inspection pouvant être utilisés pour vérifier la conformité ou prévenir la non-conformité à la Loi, ces pouvoirs d'inspection étant conformes aux autres lois fédérales.

\section{Parties visées}

La Loi s'applique à toute entreprise, personne, organisation, alliance ou entité publique qui se livre sciemment aux activités décrites faisant intervenir des agents pathogènes humains et des toxines, sauf en cas d'exemption.

\section{Portée}

La Loi ne s'applique pas à un agent pathogène humain ou à une toxine dans un environnement où il est naturellement présent s'il n'a pas été cultivé, recueilli ou extrait intentionnellement (p. ex. échantillons primaires dans le sang humain), ni à un médicament sous une forme posologique dont la vente est permise ou autorisée par la Loi sur les aliments et drogues (7) ou à un agent pathogène humain ou une toxine contenu dans un tel médicament. L'Agence a également intégré des exemptions particulières à l'exigence d'obtention d'un permis concernant les analyses de laboratoire, les tests diagnostiques et les soins vétérinaires, car les activités menées dans ces contextes sont considérées comme présentant un faible risque.

\section{Octroi de permis pour les agents pathogènes et les toxines}

Toute personne assujettie à la Loi doit présenter une demande de permis. Quatre types de permis seront délivrés en fonction de la nature et du risque des activités faisant intervenir des agents pathogènes humains ou des toxines: groupe de risque 2 (incluant les prions et les toxines), groupe de risque 3 , groupe de risque 4 et toxines classées $A B C S E$. Le titulaire de permis (ou la personne à laquelle un permis a été délivré en vertu de la $L o i)$ sera tenu de déclarer à l'Agence divers événements, tels que les expositions ou les infections contractées en laboratoire, la possession ou la production accidentelle, le gain de fonction (5), le vol ou la perte d'un agent pathogène humain ou d'une toxine. La conformité à la Norme canadienne sur la biosécurité (6), qui définit la norme minimale pour l'utilisation sûre et le confinement sécuritaire en laboratoire des agents pathogènes pour les humains, des agents pathogènes pour les animaux et des toxines, constituera une condition commune à l'octroi du permis.

D'autres exigences et conditions pour l'octroi du permis atténueront les risques inhérents que présentent des agents pathogènes ou des toxines particuliers ou à risque plus élevé. Par exemple, les personnes ayant en leur possession des agents pathogènes ou des toxines en quantité supérieure au seuil quantitatif défini devront obtenir une habilitation de sécurité conformément à la Loi. Le seuil quantitatif correspond à la quantité minimale au-delà de laquelle une toxine réglementée par la Loi est considérée comme une « toxine précisée » et, par conséquent, comme un agent biologique à cote de sécurité élevée (4). Ces exigences supplémentaires applicables aux agents pathogènes et aux toxines à risque plus élevé permettent de garantir que l'Agence sait en permanence quelles activités de laboratoire sont menées et qui les exerce au Canada. 


\section{Exigences de déclaration}

L'expression « infection contractée en laboratoire » est couramment employée pour décrire les maladies associées à des expositions à des substances infectieuses ou à des toxines en laboratoire. Toutefois, l'expression « exposition en laboratoire » est plus juste, dans la mesure où elle inclut à la fois les infections et les intoxications (découlant de l'exposition aux toxines), qu'elles soient symptomatiques ou asymptomatiques.

Les titulaires de permis sont tenus d'informer l'Agence s'ils ont des raisons de croire qu'un incident mettant en cause un agent pathogène humain ou une toxine en leur possession a ou peut avoir causé une maladie chez une personne. Cette exigence établie par la Loi a pour but d'améliorer la rapidité d'obtention, l'exactitude et l'utilité des renseignements sur les expositions en laboratoire et les infections contractées en laboratoire qui se produisent dans les installations détentrices d'un permis au Canada. Auparavant, la déclaration des expositions et des infections contractées en laboratoire était effectuée de manière ponctuelle et sur la base du volontariat au Canada.

Les exigences relatives à la déclaration des expositions et des infections contractées en laboratoire sont décrites plus en détail dans la Norme canadienne sur la biosécurité (6) qui est entrée en vigueur le $1^{\text {er }}$ décembre 2015. Elles comprennent la soumission d'un formulaire de notification de l'exposition et d'un formulaire de suivi de l'exposition au moyen du même portail électronique que celui utilisé pour les demandes de permis. Le formulaire de notification de l'exposition doit être soumis dès qu'il est raisonnablement possible de le faire et comprend des renseignements préliminaires sur la nature de l'incident et sur l'agent pathogène ou la toxine en cause. Le formulaire de suivi de l'exposition (qui doit être soumis dans les 15 jours suivant la notification de l'exposition si un ABCSE est en cause ou dans les 30 jours suivant la notification de l'exposition si un agent pathogène humain ou une toxine non classé ABCSE est en cause) fournit des précisions supplémentaires sur la ou les expositions et sur les résultats de l'enquête, notamment sur les causes fondamentales associées à l'incident.

La communication rapide d'information dans un formulaire de notification de l'exposition permettra à l'Agence de surveiller l'apparition de tendances en temps réel et de déclencher l'émission d'avis de biosécurité s'il y a lieu. Les renseignements plus détaillés fournis dans le formulaire de suivi de l'exposition permettront d'apporter des modifications ou des mises à jour aux pratiques exemplaires et à la formation en matière de biosécurité, ainsi que de bâtir une base de données probantes pouvant être analysées à l'échelle nationale pour éclairer les directives actuelles et futures sur le bioconfinement et la biosécurité.

Au fil du temps, l'évolution vers une déclaration continue et systématique par le biais d'un système de surveillance fournira des données fiables permettant d'établir des dénominateurs pour la population vulnérable (dans l'ensemble, par secteur, par types de travail, etc.), d'évaluer les expositions (de manière prospective), d'estimer les taux d'incidence des expositions et des infections contractées en laboratoire, de fournir des données en temps réel aux fins d'atténuation et de prévention, et d'établir une base solide de données probantes pour faciliter la prise de décisions visant à améliorer les politiques, la planification et la formation.

\section{Surveillance des tendances}

L'Agence évaluera les données de surveillance en continu afin de surveiller les tendances, documenter les notifications et avis de biosécurité et les pratiques d'inspection en fonction du risque, de participer à l'élaboration de pratiques exemplaires en matière de biosûreté, d'introduire les mesures de suivi de l'Agence lorsqu'elles sont nécessaires et de contribuer aux données et aux connaissances nationales et internationales sur les incidents d'exposition et d'infection contractée en laboratoire.

Les renseignements produits par le système national de déclaration des incidents seront communiqués aux intervenants de l'ensemble de la communauté de pratique en laboratoire au moyen d'un rapport annuel sur les tendances nationales agrégées. Ce rapport résumera les principaux résultats sur les incidents d'exposition et d'infection contractée en laboratoire ainsi que les conclusions des enquêtes. Étant donné 
qu'il sera nécessaire de recueillir des données pendant plusieurs années pour pouvoir établir un niveau de référence fiable à des fins de comparaison statistique, les premiers rapports annuels présenteront une description du système de déclaration et des taux de déclaration parmi les titulaires de permis, d'établir des cadres d'analyse (graphiques et tableaux) et de résumer les leçons tirées et les points saillants sur les incidents. Une fois qu'un niveau de référence fiable aura été établi, les tendances par rapport à cette estimation donneront une image plus précise de la fréquence et des facteurs contributifs des incidents, notamment des expositions à haut risque et des infections contractées en laboratoire au Canada. Ainsi, ces données contribueront à une prise de décision fondée sur des données probantes pour guider les pratiques actuelles et futures en matière de bioconfinement et de biosûreté.

\section{Discussion}

Grâce à la mise en application de la Loi et du Règlement, l'Agence est en bonne position pour atténuer les risques de libération accidentelle ou intentionnelle d'un agent infectieux ou d'une toxine, et donc de propagation d'une maladie transmissible. L'Agence saura qui a en sa possession quels agents pathogènes humains et quelles toxines au Canada, elle sera en mesure de mettre en œuvre des mesures de contrôle de la sûreté et de la sécurité pour tous les laboratoires manipulant des agents pathogènes humains et des toxines, qu'ils soient importés ou créés au Canada, et elle disposera d'un processus rigoureux d'évaluation de la sécurité ainsi que d'exigences rendant obligatoire la déclaration des incidents.

\section{Conclusion}

Par la mise en application de la Loi et du Règlement, le régime de contrôle des agents pathogènes humains non seulement prône la sûreté et la sécurité dans les laboratoires, mais il permet également aux laboratoires de santé publique canadiens d'intervenir rapidement en cas d'éclosion de maladie, il favorise les pratiques scientifiques exemplaires les plus novatrices dans les universités et les instituts de recherche canadiens et maintient un avantage compétitif pour les entreprises canadiennes.

\section{Remerciements}

Le Centre de la biosûreté tient à remercier pour leur contribution les intervenants canadiens qui ont aidé à l'élaboration d'un cadre national de supervision et de réglementation qui s'efforce de laisser la place à l'innovation, au progrès et à la croissance, tout en maintenant une sûreté et une sécurité optimales en ce qui concerne les agents pathogènes. Le temps précieux qu'ils nous ont consacré et les idées partagées pendant une série de consultations intersectorielles organisées depuis 2010 ont contribué à bâtir un cadre global de contrôle des agents pathogènes et des toxines et les programmes à l'appui qui ont renforcé les systèmes de gestion de la biosûreté visant à protéger la santé publique au Canada.

Nous souhaitons remercier également notre groupe multidisciplinaire de collègues, aussi bien à l'interne qu'en dehors du Centre de la biosûreté et de l'Agence. Tout particulièrement, les conseils, le soutien et la contribution continue de nos collègues du ministère de la Justice et de l'Agence canadienne d'inspection des aliments ont été essentiels pour permettre la mise en application complète de la Loi et du Règlement.

En nous tournant vers l'avenir, nous sommes reconnaissants pour la préparation de notre réseau d'intégration des titulaires de permis et des agents de la sécurité biologique, ainsi que pour leur engagement permanent à garantir le plus haut niveau de sûreté et de sécurité à l'égard des agents pathogènes au Canada.

\section{Conflit d'intérêts}

Aucun. 


\section{Références}

(1) Federal Bureau of Investigation (FBI). Famous cases and criminals: Amerithrax or Anthrax investigation . Washington DC: The FBI; no date. https://www.fbi.gov/about-us/history/famous-cases/anthrax-amerithrax.

(2) Centers for Disease Control and Prevention (CDC). SARS coronavirus infection in Singapore patient. Atlanta GA: CDC; 2005.

http://www.cdc.gov/sars/media/Singapore-2003-09-18.html.

(3) Centers for Disease Control and Prevention (CDC). Human Salmonella Typhimurium infections associated with exposure to clinical and teaching microbiology laboratories (Final update). Atlanta GA: CDC; 2012. http://www.cdc.gov/salmonella/2011/lab-exposure-1-17-2012.html.

(4) Government of Canada. Human Pathogens and Toxins Act (HPTA). S.C. 2009,c.24.

(Disponible en français : http://laws-lois.justice.gc.ca/fra/lois/H-5.67/page-1.html).

(5) Government of Canada. Human Pathogens and Toxins Regulations (HPTR). SOR/2015-44 October 27, 2015. (Disponible en français : http://gazette.gc.ca/rp-pr/p2/2015/2015-03-11/html/sor-dors44-fra.php).

(6) Government of Canada. Canadian Biosafety Standard, 2nd edition. Ottawa ON: Government of Canada; March 11, 2015.

(Disponible en français : http://canadianbiosafetystandards.collaboration.gc.ca/cbs-ncb/index-fra.php).

(7) Government of Canada. Food and Drugs Act. (R.S.C., 1985, c. F-27).

(Disponible en français : http://laws-lois.justice.gc.ca/fra/lois/f-27/). 